$1-1-2004$

\title{
Theoretical modeling of an A6 relativistic magnetron
}

\author{
D. J. Kaup \\ University of Central Florida
}

Find similar works at: https://stars.library.ucf.edu/facultybib2000 University of Central Florida Libraries http://library.ucf.edu

This Article is brought to you for free and open access by the Faculty Bibliography at STARS. It has been accepted for inclusion in Faculty Bibliography 2000 s by an authorized administrator of STARS. For more information, please contactSTARS@ucf.edu.

\section{Recommended Citation}

Kaup, D. J., "Theoretical modeling of an A6 relativistic magnetron" (2004). Faculty Bibliography $2000 s$. 4478.

https://stars.library.ucf.edu/facultybib2000/4478

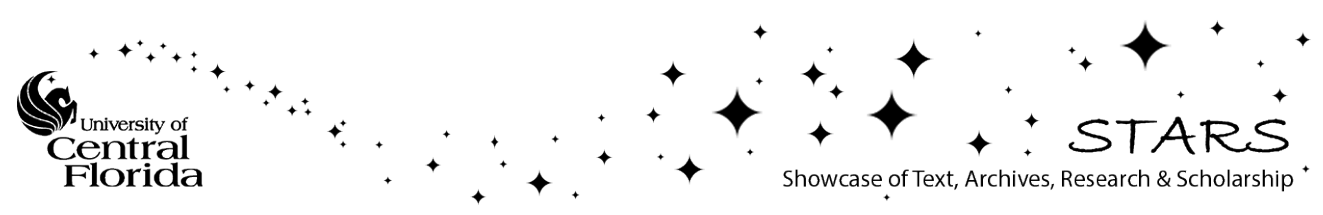




\title{
Theoretical modeling of an $\mathbf{A} 6$ relativistic magnetron
}

\author{
D. J. Kaup ${ }^{\mathrm{a})}$ \\ University of Central Florida, Orlando, Florida
}

(Received 27 August 2003; accepted 9 February 2004; published online 11 May 2004)

\begin{abstract}
The analytical modeling of the initialization stage of a relativistic magnetron of the A6 cylindrical design is presented, where only two dominant modes are used: a direct current (dc) background mode and a radio frequency (rf) pump mode. These two modes interaction nonlinearly, with the dc background being driven by the dc electromagnetic forces and the ponderomotive forces of the rf mode, while the rf mode is the most unstable linear eigenmode on this dc background. In cylindrical geometry, the diocotron resonance is found to occur over a broader region than in planar models. In fact, in certain parameter regimes, the resonance can appear twice, once near the Brillouin edge, and second, just below the anode. In these parameter regimes, the oscillating electrons can be accelerated twice. Numerical results for the initiation stage agree quite well with the known experimental results on the A6. Results for $350 \mathrm{kV}$ are emphasized, and similar results have also been obtained for voltages between 300 and $500 \mathrm{kV}$. Numerical data are presented that indicate a possible source for a nonlinear instability, which could give rise to pulse shortening, in the later operating stage, where the device should be smoothly delivering power. (C) 2004 American Institute of Physics. [DOI: 10.1063/1.1710518]
\end{abstract}

\section{INTRODUCTION}

The analytical modeling of crossed-field vacuum devices, such as crossed-field amplifiers (CFAs) and magnetrons, can give new insights into their operation, and the physical processes that occur inside them. These devices have been analyzed with "particle-in-cell" (PIC) codes (see Refs. 1-4) and guiding center theory (see Refs. 5-8, and references therein). Our modeling approach is complementary, in that we use Fourier modes. The original Fourier multiscale expansion for a planar magnetron model was first described in Ref. 9, and the importance of the second-order (quasilinear) conditions for determining the operating electron density profiles was detailed in Refs. 10 and 11. This approach produces reasonable predictions for the phase shifts, dc current flow, and other characteristics of these devices. ${ }^{12}$ Furthermore, when the total density is reconstructed from the high-frequency rf wave and the dc background, the resulting density profiles are similar to those seen in numerical simulations, showing the well-known spoke structure (see Fig. 2 below).

Any strong rf electric field propagating in the slow wave structure of a crossed-field, electron vacuum device will drive a Brillouin sheath ${ }^{13}$ unstable $e^{10,11}$ by means of a Rayleigh instability, ${ }^{14}$ whenever a wave-particle resonance (diocotron) occurs inside the sheath. This linear wave-particle instability originates in a Rayleigh-like equation ${ }^{11}$ with a shear flow. Once this linear instability initializes, the laminar flow of the electrons is strongly disturbed, and a nonlinear instability is then triggered, which is a second-order diffusion process. ${ }^{9,15,16}$ This nonlinear diffusion process causes the electrons to redistribute into a new average background

\footnotetext{
a) Telephone: 407-823-2795: fax: 407-823-6253. Electronic mail: kaup@ucf.edu
}

dc density profile, one which will be in equilibrium with the nonlinear diffusion process, which is driven by the ponderomotive pressure of the propagating if wave. ${ }^{10,11}$ This diffusion process is driven by the density gradient at the edge of the sheath, ${ }^{10}$ which for a Brillouin flow is very large. The total profile, consisting of the new stationary dc background and the rf oscillations, appears as an oscillating background with a series of periodic "spokes," as seen in numerical simulations with PIC codes. ${ }^{1,2,6}$ These spokes carry the dc current from the cathode to the anode. The physics of the turn-on process for developing these spokes has been described in Ref. 12.

Previous studies of the A6 have included the study of relativistic planar models ${ }^{6,17-19}$ and also the observation that there is an instability which could arise whenever the dc radial current becomes too large. ${ }^{20}$ Studies by others have included the derivation of the dispersion relation and the growth rates of the diocotron instability for a relativistic planar magnetron in the guiding center approximation for a tenuous beam. ${ }^{21}$ Also, the importance of the $\mathrm{rf}$ induced $E$ $\times B$ drift velocity in reducing the efficiency of such a device has been treated by Riyopoulos ${ }^{22}$ in the guiding center approximation.

Here we use analytical modeling to present an analytical and a numerical treatment of the relativistic cylindrical magnetron in the A6 configuration. In carrying out this study, our primary purpose will be to devise an analytical model, whereby one can expose and understand the physical processes occurring inside the magnetron. Thus we concentrate first on the analytics, selecting from the equations, in a consistent fashion, those terms which would be dominant. The major assumption that we use is that the dominant terms are those that come from the dc background and a single rf mode. ${ }^{9,23}$ Secondary assumptions are also used, where appropriate, to complement this one. Once the analytical results 
are obtained, then we concentrate on numerics. In this manner, one is able to reveal features about these devices that cannot be easily accessed by other methods. We have been able to outline the operating range of the parameters and give the various characteristics at any operating point, such as the phase shifts, current flow, growth rates, and power flow. ${ }^{12,23}$ We can also study the various physical processes which occur inside the tube, noting features that may be useful in the design of these devices. ${ }^{23,24}$

In Sec. II, we discuss the relativistic equations for an A6 configuration. Then in Sec. III, we discuss the equations determining the dc background solution, provided the density profile is known. In Sec. IV, we do the same for the rf solution. It is in Sec. V where we will describe how one can obtain the density profile, by use of the second-order nonlinear diffusion equation. Here we impose the adiabatic approximation and use it to determine how the density profile reshapes.

To clarify what we mean by the adiabatic approximation, we briefly describe it here. First, the dc density profile is determined by a nonlinear (quasilinear) diffusion equation, first detailed by Davidson. ${ }^{15}$ This equation consists of the sum of three basic terms. Letting $n_{0}$ be the dc density profile, $D$ the nonlinear diffusion coefficient (which is proportional to the rf power), $\tau$ a slow time, and $S$ a source term (nonlinear in $n_{0}$ ), then this equation is of the basic form $\partial_{\tau} n_{0}$ $+\partial_{r}\left(D \partial_{r} n_{0}\right)+S=0$. As is well known, diffusion equations will generally evolve so that the contributions from the first term, $\partial_{\tau} n_{0}$, tend to vanish. In this case, we have the major balance occurring between the nonlinear diffusion term and the source term. The adiabatic approximation is where we assume that this balance is the key balance, so that we then ignore the first term. As to why this balance occurs, we note that as the device is initiated, due to the relatively slow (to the electrons) changes in the dc voltage, one would expect the electrons to have time to diffuse and reshape the density profile. Thus one would expect the transient states to be small, and therefore the density profile, at every instant of time, should be essentially an equilibrium state, as determined by the stationary solutions of this nonlinear diffusion equation. This form of modeling is shown to produce reasonable predictions for the behavior of these devices.

Once we obtain the analytical results, then we turn to numerics to evaluate the solutions of these equations. One feature of these equations is that they are "stiff" near the Brillouin edge, requiring care in varying the parameters and the initial values. At the same time, one has to determine four internal parameters, whose values are essential in obtaining the correct dc and rf solutions, as well as to match to the applied voltage and external ambient magnetic field. How this can be done by an iteration procedure is described in Sec. VI. Our general results will be presented mostly in graphical form in Sec. VII, and concluding remarks will be presented in Sec. VIII.

\section{BASIC EQUATIONS AND APPROACH}

The geometry and configuration that we shall be using is shown in Fig. 1, which is a representation of the A6. ${ }^{25}$ In the

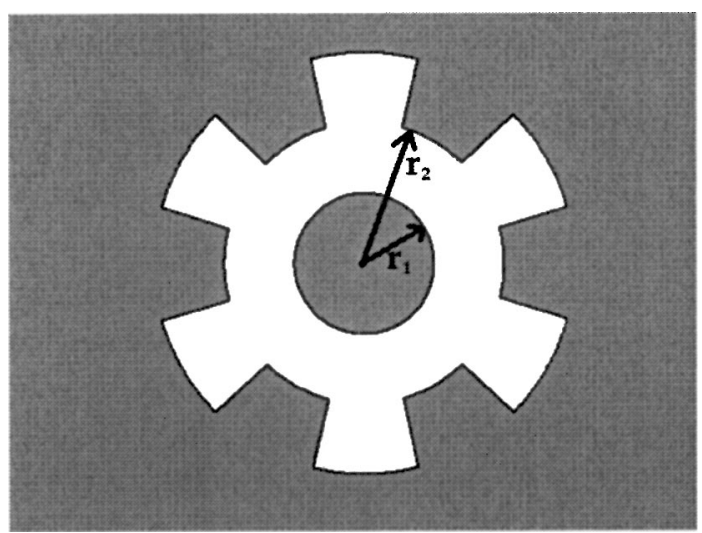

FIG. 1. A representation of the geometry of a cylindrical magnetron. The cathode is the surface of the inner shaded area at $r=r_{1}$ and the anode is at $r=r_{2}$, which is the inner surface of the vanes in the vane-slot slow-wave structure surrounding the cathode. The sheath will be formed just outside the cathode surface. The $z$ direction is out of the paper.

middle is the cathode, located at $r=r_{1}$. We take the external ambient dc magnetic field to be of strength $B_{\text {ext }}$, and aligned along the negative $z$ axis (into the paper), which gives the electron drift velocity to be in the negative $\phi$ direction. Next to the cathode will be an electron sheath (not shown) which will extend out toward the anode, located at $r=r_{2}\left(>r_{1}\right)$. The anode is composed of a slow wave structure (SWS) consisting of vanes and slots, which is simply a collection of wave guides, inside of which the rf wave will resonate and grow.

To model the A6, it is necessary to perform a fully relativistic treatment of the system in cylindrical geometry. We start with the relativistic cold fluid equations for a single species, which are

$$
\begin{aligned}
& \partial_{t} n+\boldsymbol{\nabla} \cdot(n \boldsymbol{v})=0, \\
& \partial_{t}(\gamma \boldsymbol{v})+(\boldsymbol{v} \cdot \boldsymbol{\nabla})(\gamma \boldsymbol{v})+\mathbf{E}+\boldsymbol{v} \times \mathbf{B}=0,
\end{aligned}
$$

and Maxwell's equations,

$$
\begin{aligned}
& \boldsymbol{\nabla} \times \mathbf{E}+\partial_{t} \mathbf{B}=0, \\
& \boldsymbol{\nabla} \times \mathbf{B}-\frac{1}{c^{2}} \partial_{t} \mathbf{E}=-\frac{1}{c^{2}} n \boldsymbol{v},
\end{aligned}
$$

with the initial conditions

$$
\begin{aligned}
& \boldsymbol{\nabla} \cdot \mathbf{B}=0, \\
& \boldsymbol{\nabla} \cdot \mathbf{E}=-n .
\end{aligned}
$$

In the above, we have taken $n$ to be the electron plasma frequency squared $\left(4 \pi e^{2} \rho / m\right)$ where $e(m)$ is the magnitude of the electronic charge (mass) and $\rho$ is the electron number density. $E$ is $\mathrm{e} / \mathrm{m}$ times the electric field, and $B$ is $e /(m c)$ times the magnetic field. Thus the units of $E$ are acceleration, and the unit of $B$ is frequency. We will take the fluid velocity vector to be always in the $r \phi$ plane, and all quantities (except one) to be independent of the $z$ coordinate. The one exception is the second-order dc contribution to the $\phi$ component of the second-order dc magnetic field, which, as we shall see, must be taken to be linear in $z$. This follows from 
Ampère's law and the presence of the second-order $r$ component of the dc current density. $\gamma$ is the standard relativistic factor, $1 / \sqrt{1-\boldsymbol{v} \cdot \boldsymbol{v} / c^{2}}$.

It is from these equations (1)-(6), the geometry, an expansion in dominant modes, the specified parameters, and simplifying assumptions that all our results will follow. As we have done before, ${ }^{23}$ we assume that we will only need to consider one oscillating rf mode and the dc background mode, on which the rf mode propagates. This will be a basic model, which one could further refine as the need arises. To simplify matters, we will assume that we will not need to use Floquet theory, although the system is periodic in the azimuthal direction (see discussion on this point below). Thus we take the dc solution to be cylindrically symmetric and the rf mode to be a single mode of the form $e^{i(m \phi-\omega t)}$, where $m$ is the azimuthal modal number and $\omega$ is a complex frequency, with the real part being the rf oscillation frequency and the imaginary part $(\operatorname{Im} \omega=\sigma)$ being the growth rate $\sigma$. At the same time, we will allow for a slow-time dependence in the zeroth-order dc terms, since these quantities will have to slowly shift in order to accommodate, and adjust to, any growing rf mode. This expansion is primarily an expansion in terms of Fourier components, one component (dc) which is independent of $\phi$ and the fast time, and one component of the form $e^{i(m \phi-\omega t)}$. We will later expand the dc component in a simple multiple-time scale with only two scales: a constant part and a slow-time part. ${ }^{9}$ Thus the general form of this expansion, for any quantity $G$, is

$$
G=G_{0}(r, \tau)+\epsilon G_{1}(r) e^{i(m \phi-\omega t)}+\epsilon^{2} G_{2}(r) e^{2 \sigma t},
$$

where $\tau$ is the slow time and the expansion parameter is $\epsilon$. In line with the adiabatic approximation, we will assume that the derivation of any zeroth order quantity $G_{0}$, with respect to $\tau$, will always be small compared to the ponderomotive and gradient terms.

Here we will only consider the initialization of the device. One starts with an external magnetic field applied across the device, and then the dc voltage is turned on, slowly compared to the electron cyclotron frequency. The electron sheath forms with an approximate cylindrically symmetric, stationary Brillouin flow. As the dc voltage increases, the Brillouin shear flow eventually becomes strong enough to excite the linear wave particle (diocotron) resonance $\left(\mathcal{R} \omega / m=v_{\text {drift }} / r\right)$, where $\mathcal{R} \omega$ is the real part of the complex frequency $\omega$. At this point, a growing linear instability initializes. As it grows, due to nonlinear terms in the equations, it will beat against itself and create ponderomotive-like forces, which become additional contributions to the dc components of the equations. These forces cause shifts in the dc quantities, proportional to the square of the rf amplitudes, as well as determine the shape of the electron density profile.

In regard to Floquet theory, we make the following comment. For the A6, Floquet theory would require that we couple all modes where the $m$ 's differed by $\pm 6 j$, where $j$ is any integer. However, as long as one is not near an edge of a
Brillouin zone (the $2 \pi$ mode is at such an edge, the $\pi$ mode is midway between edges), any Floquet solution would generally be dominated by one of these modes. We therefore assume that the Floquet solution is indeed dominated by one of these modes, and that modes differing by $\pm 6 j$ from that mode may be ignored. For the A6 and our geometry, the $m$ $=-3$ mode is the $\pi$ mode, for which this approximation would work best. We also expect it to be a good approximation for the two adjacent modes, $m=-2$ and $m=-4$, but possibly poor for the $m=-1$ and $m=-5$ modes.

\section{BACKGROUND SOLUTION}

We begin our analysis by inserting an expansion of the form (7), for all dependent variables, into the relativistic cold-fluid, Maxwell equations, and expand the equations. We note that $B_{2 \phi}$ must be linear in $z$, in order to satisfy Ampère's law. As mentioned earlier, the zeroth-order terms will all be generally dependent on the slow-time scale, $\tau=\epsilon^{2} t$.

The equations will decompose into coefficients of various powers of $e^{i m \phi}$ and $\epsilon$. We solve iteratively, starting at the lowest power of $\epsilon(=0)$. The $\epsilon=0$ terms of each equation (1)-(6), gives us the conditions for the existence of the "background" or dc solution. ${ }^{16}$ Detailing these, from Newton's equations, we obtain a quadratic relation for the drift velocity,

$$
E_{0}-\gamma_{0} \frac{v_{0}^{2}}{r}+v_{0} B_{0}=0
$$

where it is understood that the zeroth-order quantities given above have the appropriate component for that field. ( $E_{0}$ is the radial component, $v_{0}$ is the $\phi$ component, and $B_{0}$ is the $z$ component). From Poisson's law, given the density, the dc electric field is given by

$$
\frac{1}{r} \partial_{r}\left(r E_{0}\right)=-n_{0}
$$

and from Ampère's law,

$$
\partial_{r} B_{0}=-n_{0} v_{0} / c^{2}
$$

we can determine the radial dependence of $B_{0}$. We take the dc electric field to vanish at the cathode (the space-charge limited current condition) and the average of the dc magnetic field over the interaction region to be equal to the externally applied dc magnetic field $B_{\text {ext }}$. The latter also conserves the magnetic flux through the interaction region. These conditions are

$$
E_{0}\left(r_{1}\right)=0, \quad \int_{r_{1}}^{r_{2}} r B_{0} d r=\left(r_{2}^{2}-r_{1}^{2}\right) B_{\text {ext }} .
$$

A third condition is that the electric field must give the applied dc voltage, 


$$
-\int_{r_{1}}^{r_{2}} E_{0} d r=V_{\mathrm{dc}}
$$

We shall later need the derivative of $v_{0}$ with respect to $r$. This can be obtained by differentiating (8) and using (9) and (10) to eliminate the derivatives of $B_{0}$ and $E_{0}$. That results in

$$
\partial_{r} v_{0}=\frac{r n_{0} \gamma_{0}^{2}-v_{0} B_{0}}{r D_{B 0}}
$$

where the quantity $D_{B 0}$ is

$$
D_{B 0}=B_{0}-\frac{\gamma_{0} v_{0}}{r}\left(1+\gamma_{0}^{2}\right)
$$

We solve this system by taking (13) to define $v_{0}$, with $v_{0}=0$ at the cathode (this is equivalent to the space-charge limited current condition), and the integral of (10) to define $B_{0}$. Its value at the cathode will be adjusted so that the integral in (11) is satisfied. Then we use (8) to evaluate $E_{0}$. If the denominator in (13) ever becomes zero at some value of $r$ in the interaction region, then this corresponds to an unallowable state, since $v_{0}$ must remain finite. This condition never occurs in a planar system (where $r=\infty$ ). In a cylindrical system, in order to have magnetic insulation, one must use a stronger magnetic field than in the planar case, because in addition, one must also overcome the centrifugal force on the electrons. The condition $D_{B 0}>0$ insures that any additional magnetic field, required to counteract the centrifugal force and to maintain magnetic insulation, will be present.

\section{THE rf SOLUTION}

Now we turn our attention to the first-order (rf) solutions. These are the linearized equations, and have been given before. ${ }^{9,17,18,26-28}$ An important point in the consideration of the form that we shall use is that it is the particle dynamics that are the most important and fundamental. Consequently, we have found that it is best to eliminate the electromagnetic fields in favor of the velocities,,${ }^{9,27}$ or equivalently, in terms of Lagrangian displacements. ${ }^{14,18,29}$ For this system, the first-order Lagrangian displacements can be defined by

$$
v_{1 r}=-i \omega_{e} \xi_{r}, \quad v_{1 \phi}=-i \omega_{e} \xi_{\phi}+\left(\frac{v_{0}}{r}-\partial_{r} v_{0}\right) \xi_{r}
$$

where $\omega_{e}=\omega-m v_{0} / r$, which is the Doppler shifted frequency seen in the moving electron's frame, $\boldsymbol{\xi}$ is the firstorder Lagrangian displacement, and the subscript 1 will generally refer to a first-order quantity.

Of the rf components of the electromagnetic fields, two components of the rf magnetic field are found to vanish $\left(B_{1 r}=0=B_{1 \phi}\right)$. We also take the $z$ components of the electric field, the velocity, and the Lagrangian displacement to vanish $\left(E_{1 z}=0=v_{1 z}=\xi_{z}\right)$, so that the $\mathrm{rf}$ motion will remain in the $r-\phi$ plane. The rf component of the number density is given by

$$
n_{1}=-\frac{1}{r} \partial_{r}\left(r n_{0} \xi_{r}\right)-i \frac{n_{0} \xi_{\phi}}{r}
$$

and the nonzero electromagnetic field components are given by

$$
E_{1 \phi}=\gamma_{0}^{3} \xi_{\phi} \omega_{e}^{2}-i D_{B 0} \xi_{r} \omega_{e}
$$

$$
\begin{aligned}
B_{1 z}= & -\frac{\omega}{c^{2} m_{e}}\left[r \gamma_{0} \omega_{e}^{2}-2 D_{B 0} v_{0}-\gamma_{0}\left(1+\gamma_{0}^{2}\right) v_{0}^{2}\right. \\
& \left.+n_{0} v_{0} m_{e}\right] \xi_{r}-\frac{i r \omega D_{B 0} \omega_{e}}{c^{2} m_{e}} \xi_{\phi},
\end{aligned}
$$

where

$$
\begin{aligned}
& m_{e}=m-\omega \frac{r v_{0}}{c^{2}}, \\
& \omega_{e}=\omega-m \frac{v_{0}}{r} .
\end{aligned}
$$

Due to the combination of the relativistic factor and the cylindrical symmetry, the analytical expressions for the equations of motion becomes essentially too complex for hand calculations. Both the planar relativistic case ${ }^{17,19}$ and the cylindrical nonrelativistic case ${ }^{30}$ have been obtained by hand. However, the combination of relativistic plus cylindrical increases the complexity by an exponential factor. Consequently, from this order on, we have resorted to using the symbolic computational software MACSYMA (Ref. 31) to obtain the expansion of, and the reduction of, these equations. By taking either the nonrelativistic limit, or the planar limit, one can verify that these equations do have the proper limiting forms, and therefore can verify these equations in those limits. We have done this for the planar limit, and do find agreement with the previous planar equations. ${ }^{23}$ We shall present no derivation here, and shall simply present the final reduced results.

The first-order equations, with the aid of MACSYMA, can be reduced to a set of second-order ordinary differential equations (ODE), of the form

$$
\begin{aligned}
& \frac{d \xi_{r}}{d r}=C_{r r} \xi_{r}+i C_{r \phi} \xi_{\phi}, \\
& \frac{d \xi_{\phi}}{d r}=i C_{\phi r} \xi_{r}+C_{\phi \phi} \xi_{\phi} .
\end{aligned}
$$

With the aid of MACSYMA, the coefficients are found to be 


$$
\begin{aligned}
& C_{r r}=-\frac{1}{r}-\frac{2 \gamma_{0}^{2} v_{0}\left(\omega_{e}+m_{e} \frac{v_{0}}{r}\right)}{m_{e} c^{2}}+\frac{\gamma_{0}^{6} v_{0}}{A r^{2}}\left[4 m_{e} \omega_{e}+\frac{v_{0}}{c^{2} r}\left(r^{2} \omega_{e}^{2}-2 \gamma_{0}^{2} v_{0}^{2}\right)\right]-\frac{\gamma_{0}^{3} v_{0}}{A m_{e} c^{2}}\left\{n_{0}\left(2 \omega_{e}+\frac{v_{0} m_{e}}{r}\right)+\frac{2 \gamma_{0}^{3} \omega_{e}}{r^{2}}\left[2 r^{2} \omega_{e}^{2}\right.\right. \\
& \left.\left.+\left(\gamma_{0}^{2}-3\right) v_{0}^{2}\right]\right\}+\frac{\gamma_{0}^{3} \omega_{e}}{c^{2} D_{B 0} m_{e} r}\left[r^{2} \omega_{e}^{2}+v_{0}^{2}\left(3 \gamma_{0}^{2}-1\right)\right]+\frac{2 \gamma_{0}^{4}}{A D_{B 0} r}\left\{\frac{\gamma_{0}^{3}\left(\gamma_{0}^{2}+1\right)}{r^{3}}\left[m_{e} \omega_{e} r-\gamma_{0}^{2} \frac{v_{0}^{3}}{c^{2}}\right] v_{0}^{2}-n_{0} m_{e} \omega_{e}\right\} \\
& +\frac{\gamma_{0}^{4}}{c^{2} A D_{B 0} m_{e} r}\left\{\omega_{e}^{3} \gamma_{0}^{3}\left[r^{2} \omega_{e}^{2}+v_{0}^{2}\left(\gamma_{0}^{2}-3\right)\right]-\frac{2 \gamma_{0}^{5}\left(1+\gamma_{0}^{2}\right) v_{0}^{6} \omega_{e}}{c^{2} r^{2}}+n_{0}\left[r^{2} \omega_{e}^{3}-v_{0}^{2} \omega_{e}\left(1+\gamma_{0}^{2}\right)+2 \gamma_{0}^{2} \frac{v_{0}^{3}}{r} m_{e}\right]\right\}, \\
& C_{r \phi}=-\gamma_{0}^{2} \frac{m_{e}}{r}+\frac{\gamma_{0}^{2} r \omega_{e}^{2}}{c^{2} m_{e}}+2 \gamma_{0}^{6}\left(1+\gamma_{0}^{2}\right) \frac{v_{0} \omega_{e}}{A r^{2}}+\frac{\gamma_{0}^{3} \omega_{e}^{2}}{c^{2} A m_{e}}\left[r n_{0}+\frac{\gamma_{0}^{3}}{r}\left(r^{2} \omega_{e}^{2}+2 \gamma_{0}^{2} \frac{v_{0}^{4}}{c^{2}}\right)\right]+\frac{\gamma_{0}^{5} v_{0}^{2} \omega_{e}}{c^{2} D_{B 0} r}-\frac{2 \gamma_{0}^{5} v_{0} \omega_{e}^{2}}{c^{2} D_{B 0} m_{e}} \\
& +\frac{\gamma_{0}^{4} \omega_{e}}{A D_{B 0} r^{3}}\left\{v_{0}^{2}\left[2\left(1+\gamma_{0}^{2}\right)+\frac{r^{2} \omega_{e}^{2}}{c^{2}}\right] \gamma_{0}^{5}-n_{0} r^{2}\left(3 \gamma_{0}^{2}-1\right)\right\}-\frac{2 \gamma_{0}^{9} \omega_{e}^{2} v_{0}}{c^{2} A D_{B 0} m_{e} r^{2}}\left[r^{2} \omega_{e}^{2}-v_{0}^{2}\left(1+\gamma_{0}^{2}\right)\right], \\
& C_{\phi r}=\frac{m_{e}}{\gamma_{0}^{2} r}\left(1-2 r \gamma_{0}^{3} \frac{n_{0}}{A}\right)-2 \frac{\gamma_{0}^{2} v_{0}^{2}}{A r^{3}}\left[\left(3 \gamma_{0}^{2}-1\right) m_{e}+\gamma_{0}^{2} \frac{r v_{0}}{c^{2}} \omega_{e}\right]+\frac{2 v_{0}^{3}}{c^{2} A r^{2} \omega_{e}}\left[2 n_{0}+\gamma_{0}^{6} \frac{v_{0}^{4}}{c^{2} r^{2}}\right]+\frac{4 v_{0} m}{\gamma_{0}^{2} m_{e} r \omega_{e}} \\
& +\frac{r^{4} \omega_{e}^{4}+3 v_{0}^{2} r^{2} \omega_{e}^{2}\left(3 \gamma_{0}^{2}-1\right)+2 v_{0}^{2}\left(\gamma_{0}^{4}-6 \gamma_{0}^{2}+1\right)}{A c^{2} \gamma_{0}^{2} m_{e} r^{3}}+\frac{n_{0} \gamma_{0}}{c^{2} A m_{e} r}\left[r^{2} \omega_{e}^{2}+\left(3 \gamma_{0}^{2}-1\right) v_{0}^{2}\right]+\frac{v_{0} \gamma_{0}}{c^{2} D_{B 0} r^{2}}\left(4 m_{e}+\frac{r v_{0}}{c^{2}} \omega_{e}^{2}\right) \\
& -\frac{1}{D_{B 0} r \omega_{r}}\left[2 n_{0}\left(2-\frac{1}{\gamma_{0}^{2}}\right)-\frac{v_{0}\left(\gamma_{0}^{4}+4 \gamma_{0}^{2}-1\right)}{r^{2} c^{2} \gamma_{0}}\right]-\frac{2 v_{0}}{c^{2} D_{B 0} m_{e}}\left\{n_{0}+\frac{\gamma_{0}}{r^{2}}\left[2 r^{2} \omega_{e}^{2}+v_{0}^{2}\left(r_{0}^{2}-3\right)\right]\right\} \\
& +\frac{\gamma_{0}^{5} v_{0}}{A D_{B 0} r^{4}}\left\{4 m_{e}\left[r^{2} \omega_{e}^{2}-\left(1+\gamma_{0}^{2}\right) v_{0}^{2}\right]-\frac{n_{0} v_{0} \omega_{e} r^{3}}{\gamma_{0} c^{2}}+\frac{v_{0} r \omega_{e}}{c^{2}}\left[r^{2} \omega_{e}^{2}-\left(1+3 \gamma_{0}^{2}\right) v_{0}^{2}\right]\right\} \\
& -\frac{2 \gamma_{0}^{5} v_{0}}{c^{2} A D_{B 0} m_{e} r^{4}}\left[r^{2} \omega_{e}^{2}-\left(1+\gamma_{0}^{2}\right) v_{0}^{2}\right]\left[2 r^{2} \omega_{e}^{2}+\left(\gamma_{0}^{2}-3\right) v_{0}^{2}+\frac{n_{0} r^{2}}{\gamma_{0}}\right]+\frac{\gamma_{0}^{4}\left(1+\gamma_{0}^{2}\right) v_{0}^{4}}{c^{2} A D_{B 0} r^{3} \omega_{e}}\left[\frac{\gamma_{0}^{3} v_{0}^{2}}{r^{2}}+n_{0}\right] \text {, } \\
& C_{\phi \phi}=-\frac{1}{r}-\frac{2 \gamma_{0}^{2} v_{0}}{c^{2} r}-\frac{4 \gamma_{0}^{2} m_{e} v_{0}}{r^{2} \omega_{e}}+\frac{2 \gamma_{0}^{2} v_{0} \omega_{e}}{c^{2} m_{e}}+\frac{\gamma_{0}^{6} v_{0}^{2}}{A r^{3}}\left[2\left(1+\gamma_{0}^{2}\right)-\frac{r^{2} \omega_{e}^{2}}{c^{2}}\right]+\frac{\gamma_{0}\left(3 \gamma_{0}^{2}-1\right) n_{0}}{A r}+\frac{2 \omega_{e} v_{0} \gamma_{0}^{6}}{c^{2} A m_{e} r^{2}} \\
& \times\left[2 r^{2} \omega_{e}^{2}-v_{0}^{2}\left(3-\gamma_{0}^{2}\right)+\frac{n_{0} r^{2}}{\gamma_{0}^{3}}\right]-\frac{\gamma_{0}\left(1+\gamma_{0}^{2}\right)^{2} v_{0}}{D_{B 0} r^{2}}+\frac{2 m_{e}}{D_{B 0} r \omega_{e}}\left[n_{0}-\gamma_{0}^{3}\left(1+\gamma_{0}^{2}\right) \frac{v_{0}^{2}}{r^{2}}\right]-\frac{r \gamma_{0}^{3}}{c^{2} D_{B 0} m_{e}}\left[\frac{n_{0}}{\gamma_{0}^{3}}\left(\omega_{e}+m_{e} \frac{v_{0}}{r}\right)\right. \\
& \left.+\omega_{e}\left(\omega_{e}^{2}+\frac{2 \gamma_{0}^{2} v_{0}^{4}}{c^{2} r^{2}}\right)\right]-\frac{2 \gamma_{0}^{7}\left(1+\gamma_{0}^{2}\right) v_{0}}{A D_{B 0} r^{4}}\left[r^{2} \omega_{e}^{2}-\left(1+\gamma_{0}^{2}\right) v_{0}^{2}\right]-\frac{\gamma_{0}^{7} \omega_{e}}{c^{2} A D_{B 0} m_{e} r}\left\{\frac{n_{0}}{\gamma_{0}^{3}}\left[r^{2} \omega_{e}^{2}-\left(1+\gamma_{0}^{2}\right) v_{0}^{2}\right]\right. \\
& \left.+\omega_{e}^{2}\left[r^{2} \omega_{e}^{2}-\left(3-\gamma_{0}^{2}\right) v_{0}^{2}\right]-2 \gamma_{0}^{2}\left(1+\gamma_{0}^{2}\right) \frac{v_{0}^{6}}{c^{2} r^{2}}\right\} \text {, }
\end{aligned}
$$

where

$$
A=\left(D_{B 0}+\gamma_{0}^{3} \frac{v_{0}}{r}\right)^{2}+\gamma_{0}^{4} \frac{v_{0}^{2}}{r^{2}}-\gamma_{0}^{4} \omega_{e}^{2} .
$$

We will need two boundary conditions for this second-order set of ODE's. The first one will come from $E_{1 \phi}$ vanishing at the cathode (conducting surface condition), and the second one will come from matching the interior plasma solution to the electromagnetic field solution in the SWS. For the latter we require that, at the anode, the ratio of $E_{1 \phi} / B_{1 z}$ in the plasma region to match the same as that in the SWS, which will be assumed to be a vacuum region. To calculate the ratio on the plasma side, we need the forms of $E_{1 \phi}$ and $B_{1 z}$, given above by (17) and (18). To calculate the ratio on the SWS side, we solve Maxwell's equations for the given vane-slot configuration, subject to standard boundary conditions on the slot surfaces. In the numerical calculations of this ratio, we have included the first three azimuthal modes in each slot.

Given these boundary conditions and the background 
density profile, for each value of $m$, one then determines the (complex) eigenvalues of $\omega$ which will satisfy the boundary conditions. In general, there can be several eigenvalues of $\omega$ for each value of $m$. While all of these would in general be initially excited and would proceed to grow at their individual growth rates, it is the one with the largest growth rate that will eventually dominate. We then search through the list of eigenvalues for that one, and that is the one that we take as the operating mode. Typically, for the parameter space that we have searched, we have found that this mode will have $m$ in the range of $-4 \leqslant m \leqslant-1$.

\section{NONLINEAR DIFFUSION-SECOND-ORDER dc}

In second order, the rf wave beats against itself, creating a ponderomotive force. This force causes a nonlinear diffusion to initiate, wherein the steep density gradient of the Brillouin flow is reduced. Due to the nonlinear terms in (1)(6), this diffusion causes the dc solution to slowly shift away from its initial solution, at a rate proportional to the square of the rf amplitude. An analysis of all such shifts in the nonrelativistic case was given in Refs. 9 and 15, along with a discussion of the evolution of the background density profile.

Although the zeroth-order solution does evolve during the nonlinear diffusion process, by using the adiabatic approximation discussed in the Introduction, we bypass the transient region and go directly to the stationary solution, wherein the electrons have redistributed themselves to be in balance with the ponderomotive and electromagnetic forces. We have shown that this nonlinear diffusion process will be generally quite rapid. ${ }^{10}$

In the fully relativistic case, there is a lag in the growth of the second-order fields (due to the finite speed of light) which is not present in the nonrelativistic case. Along with this, the strong dc radial current creates a $B_{2 \phi}$ component, linear in $z$, which then will generate small $z$ components of the velocity and electric field, proportional to $z$. Depending on the width of the device in $z$, this could become an important consideration. However, if the device is sufficiently thin, then these effects will be small and can be ignored. The only consequence of assuming a thin device and ignoring these $z$ components of $v$ and $E$ will be that the $\phi$ component of (3) will not be exactly satisfied. Also, in an actual device, the use of "end caps" tends to limit these longitudinal effects and motions.

Taking the above thin approximation, we have that the second-order dc solution will have the following features. The second-order corrections to the dc magnetic field are given by

$$
B_{2 r}=0, \quad B_{2 \phi}=\frac{B_{0 c}^{2} C_{2} z r_{2}}{c^{2} r}, \quad B_{2 z}=-\frac{1}{2 \sigma r} \partial_{r}\left(r E_{2 \phi}\right),
$$

where $B_{0 c}$ is the value of the zeroth-order dc magnetic field at the cathode, $C_{2}$ is essentially the radial dc velocity of the electrons, and $r_{2}$ is the radius of the device at the inside of the vanes (anode). (The factors of $B_{0 c}, r_{2}$, and $c$ in the expression for $B_{2 \phi}$ are there simply to normalize $C_{2}$ to a velocity.) Since the device will carry a dc radial current, one must have a nonzero $B_{2 \phi}$ component, and thus $C_{2}$ must be nonzero. This constant will reappear later as a key quantity in the nonlinear diffusion equation, where it will essentially determine the width of the Brillouin-like section of the profile.

The second-order electric field has only a $\phi$ component $\left(E_{2 r}=0=E_{2 z}\right)$, which satisfies the equation

$$
\partial_{r}^{2} E_{2 \phi}+C_{e 1} \partial_{r} E_{2 \phi}+C_{e 0} E_{2 \phi}=C_{e S},
$$

where the coefficients are

$$
\begin{aligned}
C_{e 1}= & \frac{1}{r}+\frac{n_{0} v_{0}}{c^{2} D_{B 0}}\left(1-\frac{4 \gamma_{0}^{3} \sigma^{2}}{D}\right), \\
C_{e 0}= & -\frac{1}{r^{2}}+\frac{n_{0} v_{0}}{c^{2} D_{B 0} r}\left(1-\frac{4 \gamma_{0}^{3} \sigma^{2}}{D}\right)-\frac{4 \sigma^{2}}{c^{2}}\left(1+\frac{n_{0}}{D}\right), \\
C_{e S}= & -2 \sigma Q_{A \phi}+\frac{4 n_{0} Q_{N \phi} \sigma^{2}}{c^{2} D} \\
& +\frac{2 n_{0} Q_{N r} \sigma}{c^{2} D_{B 0}}\left(1-\frac{4 \gamma_{0}^{3} \sigma^{2}}{D}\right),
\end{aligned}
$$

and where the denominator term $D$ is defined as

$$
D=\frac{1}{\gamma_{0}}\left(D_{B 0}+\gamma_{0}^{3} \frac{v_{0}}{r}\right)^{2}+\gamma_{0}^{3}\left(4 \sigma^{2}+\frac{v_{0}^{2}}{r^{2}}\right)-n_{0} .
$$

The boundary conditions on $E_{2 \phi}$ are that it must vanish at both the cathode and the anode, since it is independent of $\phi$, and both surfaces will appear to be conducting surfaces. ${ }^{32}$ Also, $Q_{A}$ is the rf part of Ampère's equation, $Q_{N}$ is the rf part of Newton's equation. These parts are given by

$$
\begin{aligned}
Q_{A r}= & \frac{n_{1}^{*} v_{1 r}}{c^{2}}+\text { c.c., } \\
Q_{A \phi}= & \frac{n_{1}^{*} v_{1 \phi}}{c^{2}}+\text { c.c., } \\
Q_{N r}= & \left\{B_{1 z}^{*} v_{1 \phi}+i \frac{m \gamma_{0}}{r} v_{1 r} v_{1 \phi}^{*}+2 \gamma_{0}^{3} v_{0} v_{1 \phi}^{*} v_{1 r} \frac{\sigma}{c^{2}}+c . c .\right\} \\
& +\gamma_{0} \partial_{r}\left|v_{1 r}\right|^{2}-3 \gamma_{0}^{3} \frac{v_{0}^{2}}{r c^{2}}\left|v_{1 r}\right|^{2} \\
& +\frac{v_{0} \gamma_{0}}{c^{2} D_{B 0}}\left|v_{1 r}\right|^{2}\left[2 n_{0}-2 \gamma_{0}^{3} \frac{v_{0}^{2}}{r^{2}}\left(1+\gamma_{0}^{2}\right)-3 \gamma_{0}^{2} \frac{v_{0}}{r}\right] \\
& +\frac{\gamma_{0}^{3}}{r}\left|v_{1 \phi}\right|^{2}\left(1-3 \gamma_{0}^{2}\right),
\end{aligned}
$$




$$
\begin{aligned}
Q_{N \phi}= & \left\{-B_{1 z}^{*} v_{1 r}+\gamma_{0}^{3} v_{1 r}^{*} \partial_{r} v_{1 \phi}-\gamma_{0}^{3}\left[\left(3 \gamma_{0}^{2}-4\right) \frac{1}{r}\right.\right. \\
& \left.-3 \frac{n_{0} v_{0}}{D_{B 0} c^{2}}+3 \gamma_{0}^{3}\left(\gamma_{0}^{2}+1\right) \frac{v_{0}^{2}}{r^{2} c^{2} D_{B 0}}\right] v_{1 r}^{*} v_{1 \phi} \\
& + \text { c.c. }\}+2 \gamma_{0}^{3} \frac{v_{0} \sigma}{c^{2}}\left(\left|v_{1 r}\right|^{2}+3 \gamma_{0}^{2}\left|v_{1 \phi}\right|^{2}\right) \\
& -\frac{2 \sigma \gamma_{0}\left|v_{1 r}\right|^{2}}{D_{B 0}\left|\omega_{e}\right|^{2}} \partial_{r} n_{0},
\end{aligned}
$$

and where the "**" indicates the complex conjugate of the quantity, and "+c.c." means to add the complex conjugate of all the preceeding terms that are contained inside that particular bracket. Continuing, from the second-order Newtonian equations, we have that the second-order changes in the velocities are given by

$$
\begin{aligned}
v_{2 r}= & -\frac{2 \gamma_{0}^{2} \sigma}{D}\left(v_{0} B_{2 z}+Q_{N r}\right)+\frac{D_{B 0}}{\gamma_{0} D}\left(E_{2 \phi}+Q_{N \phi}\right), \\
v_{2 \phi}= & -\frac{1}{D_{B 0}}\left(1-\frac{4 \gamma_{0}^{3} \sigma^{2}}{D}\right)\left(v_{0} B_{2 z}+Q_{N r}\right) \\
& -\frac{2 \sigma}{D}\left(E_{2 \phi}+Q_{N \phi}\right),
\end{aligned}
$$

$$
v_{2 z}=0 \text {. }
$$

The next and last result which follows from the secondorder dc equations is the nonlinear diffusion equation, which determines the stationary density profile. This equation, in the adiabatic approximation, where we ignore $\partial_{\tau} n_{0}$ and the slow-time derivatives of all other quantities, is

$$
D_{n l} \partial_{r} n_{0}+N_{n l} n_{0}+C_{2} B_{0 c}^{2} D \frac{r_{2}}{r}=0,
$$

where

$$
\begin{aligned}
D_{n l}= & \frac{2 \sigma\left|\xi_{r}\right|^{2}}{\gamma_{0}}\left[\left(D_{B 0}+\gamma_{0}^{3} \frac{v_{0}}{r}\right)^{2}+\gamma_{0}^{4}\left(\frac{v_{0}^{2}}{r^{2}}+4 \sigma^{2}\right)\right], \\
N_{n l}= & 2 \gamma_{0}^{2} \sigma Q_{N r}-D c^{2} Q_{A r 0}-\frac{D_{B 0} Q_{N \phi 0}}{\gamma_{0}}-\gamma_{0}^{2} v_{0} \partial_{r} E_{2 \phi} \\
& -\left(\frac{D_{B 0}}{\gamma_{0}}+\gamma_{0}^{2} \frac{v_{0}}{r}\right) E_{2 \phi} .
\end{aligned}
$$

In (35), the subscript " 0 "s on the $Q$ 's indicate that all terms proportional to $\partial_{r} n_{0}$ have been removed. [In (34), all that we did was to move any and all terms which contained the radial derivative of $n_{0}$, over into the nonlinear diffusion coefficient $D_{n l}$, which is where they are now found. Only $Q_{A r}$ and $Q_{N \phi}$ did contain such terms.] To solve Eq. (34), we must append one boundary condition, which we take to be $n_{0 a}$, which is the value of the stationary density at the anode, $r=r_{2}$. This value of $n_{0 a}$ will appear as a control parameter in our numerical solutions.

\section{NUMERICAL METHOD OF SOLUTION}

At this point, we now have a closed system of ordinarily differential equations, and we have sufficient boundary conditions to define a solution, if one exists. The zeroth-order dc equations are nonlinear. Given $n_{0}(r)$, one can integrate them from the cathode up to the anode, given the value of $B_{0 c}$, the value of $B_{0}$ at the cathode. This value is initially unknown, but one can start with an initial guess, and by using the Newton-Raphson method, rapidly converge to the correct value that will satisfy the second condition in (11). In regard to the first-order rf equations, they are linear in the rf variables, but nonlinear in the zeroth-order quantities. Given a value for the complex frequency $\omega$, the mode number $m$, and the dc solution, by requiring $E_{1 \phi}$, (17), to vanish at the cathode, then matching at the SWS, we can uniquely determine the rf solution, up to an overall normalization constant. The second-order dc equations are linear in the second-order variables, but nonlinear in the zeroth-order variables, and quadratic (nonlinear) in the rf fields. The key quantity to determine will be the stationary density profile $n_{0}$, whose determining equation is (34). Of course, inspection reveals that once one has the stationary density profile, then all the other quantities may be determined by either quadrature or differentiating and/or algebra, whence the stationary density profile is the key quantity to obtain. We have found that these profiles may be obtained by a simple iteration technique. The basin of convergence is generally quite large, unless one is near a point where the solution no longer exists.

The general iteration method that we used is as follows. One starts with some reasonable density profile $n_{0}(r)$, either a combination of a box plus a ramp, or some nearby profile, and then solve for the dc electromagnetic fields. Once one has a starting profile and the accompanying dc fields, then one proceeds to solve the rf equations (22) for the radial and azimuthal displacements, using the given boundary conditions below Eq. (27). One checks all mode numbers, -6 $<m<0$, for the most unstable mode, varying the complex value of $\omega$ until the electron plasma solution at the anode matches that of the SWS. With this, one then has the eigenvalue $\omega$, the mode number $m$, and the rf solution, up to the overall arbitrary normalization constant.

To find the normalization constant for the rf solution, we require the total density variations, dc plus the rf oscillations, as in (7), to never be negative, since a negative electron density would be unphysical. This condition then gives an upper limit to the value of the normalization coefficient, which also is the precise value that must be used, for the following reason. In the absence of the rf waves, the density profile would relax back to the Brillouin value, which has $n_{0 a}$ vanishingly small. For the rf wave to exist, then $n_{0 a}$ must be lifted off of its Brillouin value of zero, and become sufficiently large to accommodate the amplitude of the rf oscillations, without driving the total electron density negative. Turning this argument around then for a given $n_{0 a}$, one would take the amplitude of the rf density oscillations, $\left|n_{1}(r)\right|$, to be just large enough to exactly fit under the density profile $n_{0}(r)$. Examples of this will be seen in Fig. 2 in the next section. 
n vs. $r$

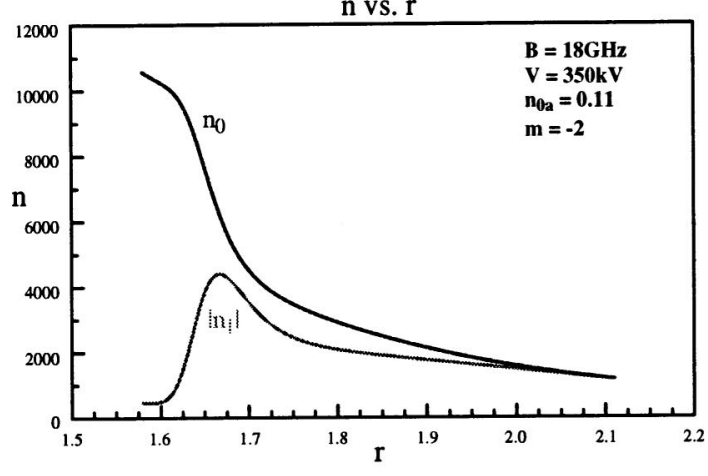

n vS. $\mathbf{r}$

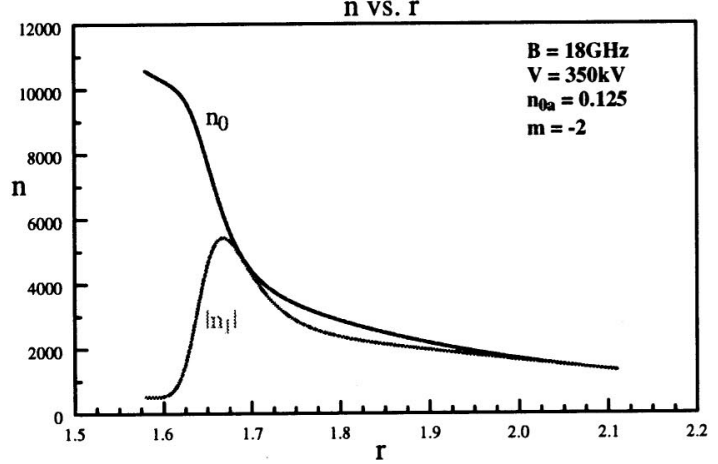

Total Density for One Period

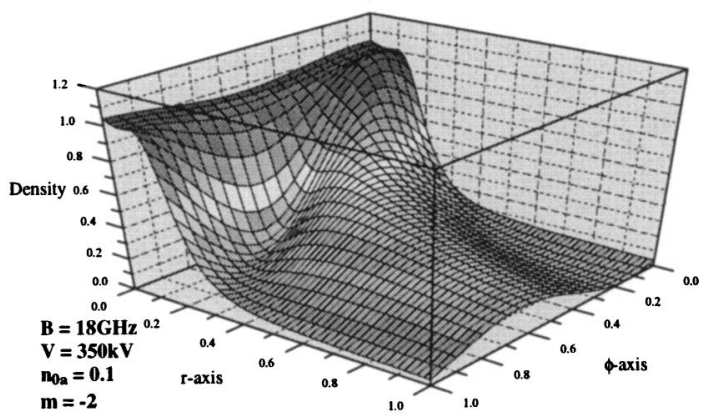

n vs. $\mathbf{r}$

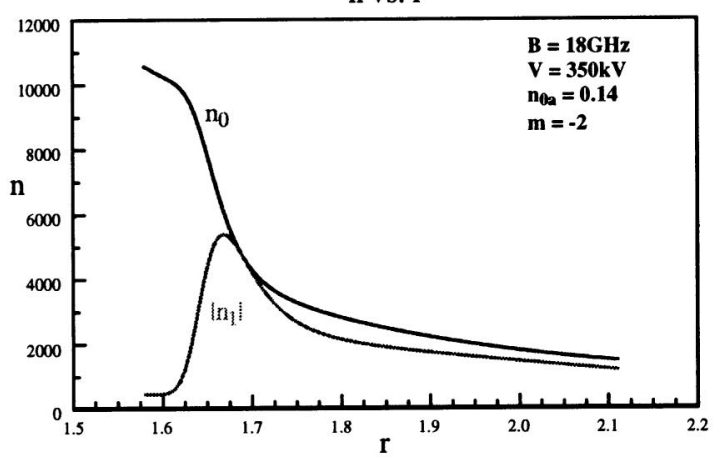

FIG. 2. Plots of certain density profiles for the A6 at $350 \mathrm{kV}$ and an ambient magnetic field of $B_{\text {ext }}=18 \mathrm{GHz}(6.4 \mathrm{~K} \mathrm{G})$ case, when the rf field is in the $m$ $=-2$ mode. In the first plot, for $n_{0 a}=0.11$, we see the density profile and the magnitude of the rf oscillations for an initiating rf field, with a peak in the rf density oscillations at the diocotron resonance. In the second plot, we have combined the dc and the rf solutions to compose the total density profile, at a slightly lower value of $n_{0 a}=0.10$. In the third plot, we show the configuration when the rf wave reaches its maximum amplitude, at $n_{0 a}=0.125$. In the final plot, we see how the configuration changes when one tries to exceed this limit. Here we have the solution for $n_{0 a}=0.14$, showing how the rf amplitude at the anode will decrease.

Once the rf normalization constant has been determined, then the rf fields are uniquely determined, up to an overall phase. Although this rf solution is consistent with the dc fields, the dc fields may not be consistent with these new rf fields. So now we turn to satisfying the condition on the density profile, given by (34), as well as calculating the second-order fields $E_{2 \phi}$ and $B_{2 z}$. The condition (34) is what is known as a "stiff" ODE. Its solution will typically have exponential behavior with very short scale lengths, on the order of the width of the edge of the classical Brillouin sheath. Furthermore, due to the stiffness of this ODE, as one varies the unknown parameter $C_{2}$, the solution can rather suddenly violate the single-particle stability limit. (In the nonrelativistic limit, the single-particle stability range for the electron density is $n_{0}<B_{0}^{2}$, which is just the Brillouin limit.) To complicate the problem further, the boundary conditions are mixed, with the value of $n_{0}$ being specified at the anode $n_{0 a}$ and other parameters being specified at the cathode.

There are probably several iteration schemes which would solve these equations. The iteration that we have used is to integrate (34) to obtain $n_{0}$, and then vary $C_{2}$, using Newton-Raphson, until the new density profile gives the correct applied dc voltage $V_{\mathrm{dc}}$. Outside of this iteration, we have another iteration whereby we vary $B_{0 c}$ until we have
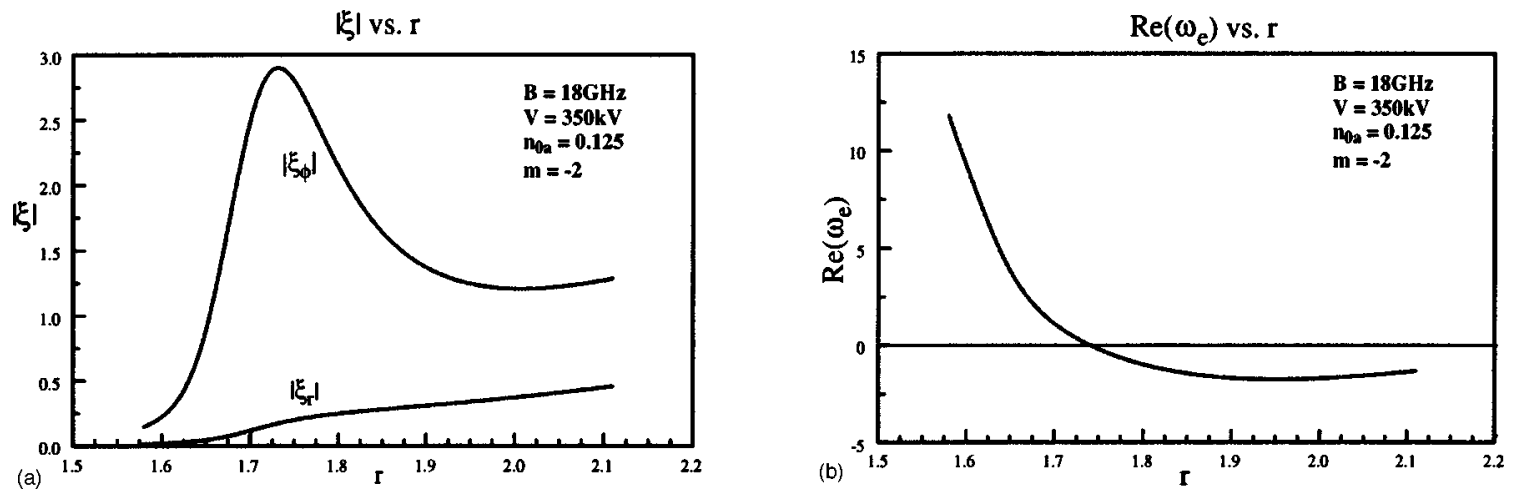

FIG. 3. In the first panel we show a plot of the magnitudes of the Lagrangian displacements, for the same parameters as Fig. 2(c). In (b), we show a plot of the real part of $\omega_{e}$ vs $r$ for the same solution. 
KEl vs. $\mathbf{r}$

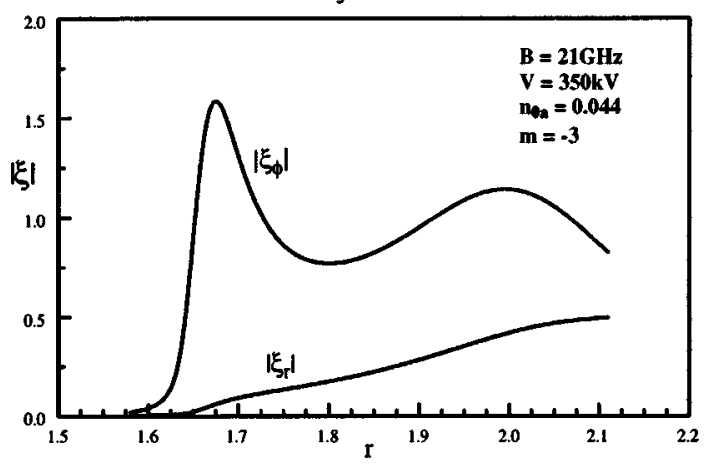

$\operatorname{Re}\left(\omega_{\mathrm{e}}\right)$ vs. $\mathbf{r}$

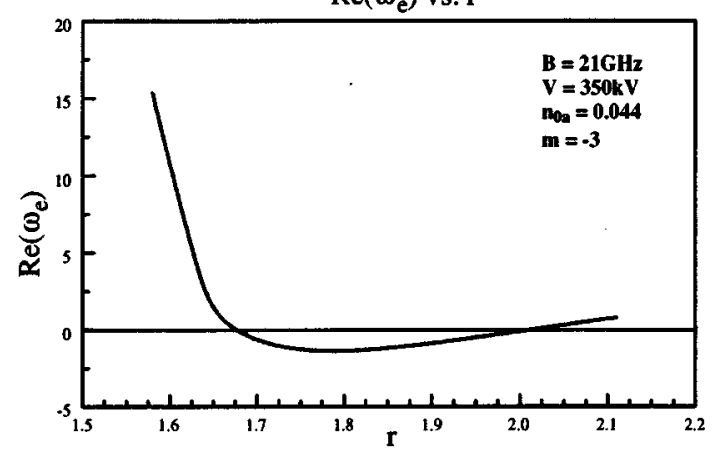

FIG. 4. Similar to Fig. 3, except for $B_{\text {ext }}=21 \mathrm{GHz}(7.5 \mathrm{~K} \mathrm{G})$ and $n_{0 a}=0.044$. This figure shows a second diocotron resonance just under the anode, and the corresponding increase in the oscillations of the azimuthal displacements.

both the correct $V_{d c}$ and $B_{\text {ext }}$. Then we use (11), (12), and (8) to determine the new values of $E_{0}, B_{0}$, and $v_{0}$ by quadrature.

Once we have the new density profile and the new dc fields, then we have the dc solution consistent with the $\mathrm{rf}$ fields. However, now the old rf fields will generally be inconsistent with the new dc fields. So we return to the calculation of the rf fields and then back to the second-order dc fields and the density profile. We continue these iterations until all the coefficients, $V_{\mathrm{dc}}, B_{0 c}, m, \omega$, and $C_{2}$, have stabilized to within a specified relative variation of no more than typically $10^{-6}$. However sometimes the convergence does not occur, and that is usually because no solution can exist for the chosen values of the parameters $\left(V_{\mathrm{dc}}, B_{\text {ext }}\right.$, and $n_{0 a}$ ). This can show up as an rf solution having a vanishing growth rate for all $m$ values, due to $V_{\mathrm{dc}}$ being too small for the given value of $B_{\text {ext }}$, or even as no solution existing for $n_{0}$ due to $n_{0 a}$ being too large, and sometimes even being too small. Occasionally we have found that the solution will converge to some period-2 solution, wherein the values of $B_{0 c}$, $m, \omega$, and $C_{2}$ converge to a repetition of alternating values. ${ }^{24}$ Period-3 and period- 4 solutions have also been seen. Thus it is not surprising that sometimes one also sees chaotic results occurring. These equations are nonlinear and little is known about necessary conditions for solutions to exist.

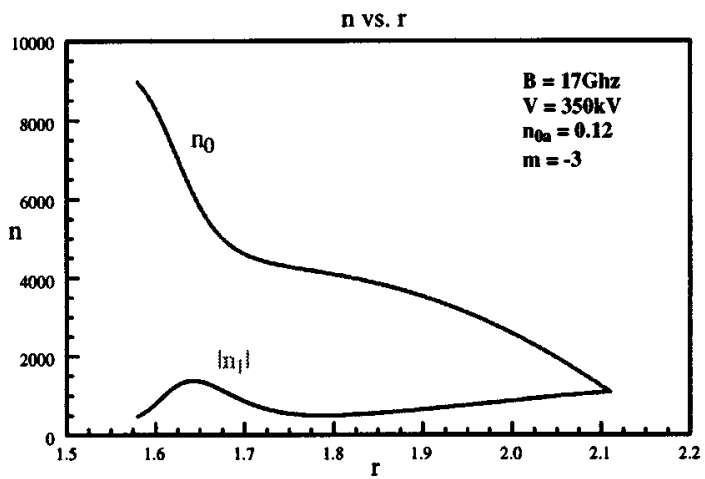

\section{NUMERICAL RESULTS}

As the first example of our results, we show in Fig. 2 a set of four typical results for the density profile $n_{0}$ and the magnitude on the rf density oscillations at $V=350 \mathrm{kV}, B_{\text {ext }}$ $=18 \mathrm{GHz}(6.4 \mathrm{~K} \mathrm{G})$, and for the mode number $m=-2$. This is toward the low side of the operational range of the magnetic field, and in this region, the density profiles tend to be "soft," and the mode numbers correspondingly tend to be low, and on the order of $-1--3$. The horizonal axis is the radius in $\mathrm{cm}$, where the cathode is on the left, starting at 1.58 $\mathrm{cm}$, and the anode is on the right, at $2.11 \mathrm{~cm}$. Figure 2(a) shows the solution when $n_{0 a}=0.11$, and we see that the $\mathrm{rf}$ field is becoming well developed, with a peak in the $\left|n_{1}\right|$ curve, which is where the diocotron resonance occurs at the edge of the sheath. [In all these figures, the units of $n$ is frequency squared, in units of $(\mathrm{rad} / \mathrm{nsec})^{2}$. However, we will give $n_{0 a}$ as a ratio compared to the square of the electron cyclotron frequency, in $\mathrm{rad} / \mathrm{nsec}$, of the external magnetic field. As such, $n_{0 a}$ is essentially just the ratio of the de density at the anode, compared to the dc density at the cathode.] As one can also see, the rf field has been scaled so that it fits entirely under the $n_{0}$ curve, and just touches it at the anode on the right. Consequently, the total density is everywhere nonnegative. In Fig. 2(b), we show a two-dimensional view

FIG. 5. The density profile, the magnitude of the rf density oscillations, and the Lagrangian displacements for a soft density profile, with $V=350 \mathrm{kV}, B_{\text {ext }}$ $=17 \mathrm{GHz}(6.1 \mathrm{~K} \mathrm{G})$, and $n_{0 a}=0.12$. 

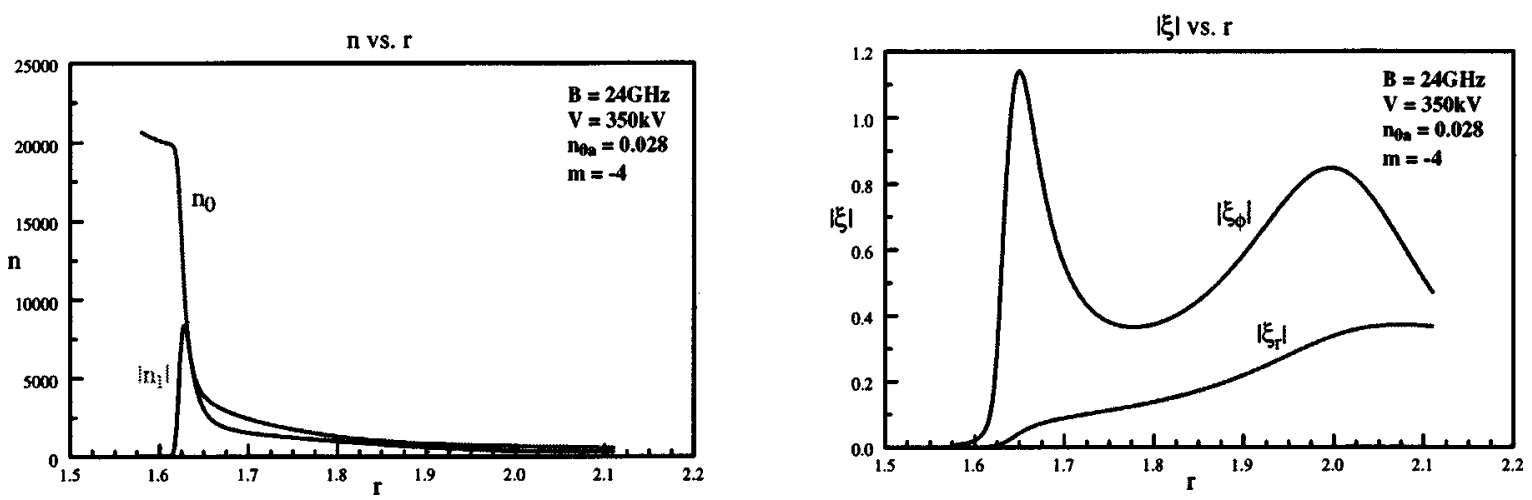

FIG. 6. The density profile, the magnitude of the rf density oscillations, and the Lagrangian displacements for a hard density profile, with $V=350 \mathrm{kV}$, $B_{\text {ext }}=24 \mathrm{GHz}(8.6 \mathrm{~K} \mathrm{G})$, and $n_{0 a}=0.028$.

for a single period of the rf wave at a slightly smaller value of the amplitude of the rf wave, $n_{0 a}=0.10$. (In these threedimensional plots, each coordinate has been scaled from zero to unity.) Here the cathode is at the back on the left, where one clearly sees the sheath. Coming out of the sheath is a spoke of electrons, reaching up to the anode, in the foreground at the right.

As the amplitude of the rf wave at the anode increases, the first thing that happens is that the value of $n_{0 a}$ will be pushed to larger values. The second thing that happens is that the diocotron peak will grow and generally narrow. Eventually, it will touch the $n_{0}$ curve, as shown in Fig. 2(c) for $n_{0 a}=0.125$. This now is effectively the largest operating amplitude that the rf wave can have at these parameters, because now, the rf amplitude becomes limited by the diocotron peak, and not the rf amplitude at the anode. An example of this is clearly shown in Fig. 2(d), which is for the slightly higher value of $n_{0 a}=0.14$. Note that now, at the anode, a gap has opened up between the background density and the rf density oscillations, with the net result that the rf oscillation amplitude has decreased at the anode. Solutions such as this latter, with the gap, are probably unphysical or unstable, since they correspond to a reduced rf amplitude of the rf wave in the vanes. These observations are in line with previous similar observations in Ref. 23.

In Fig. 3(a), we show the rf Lagrangian displacements for the $n_{0 a}=0.14$ solution. The units for each axis is in $\mathrm{cm} \mathrm{s}$. One notes the enhanced amplitude of the rf oscillations in the $\phi$ direction, at the diocotron resonance, as well as the beginning of another enhancement as one nears the anode. The latter can be understood from Fig. 3(b), where we show the real part of $\omega_{e}$ vs $r$. What is different here from the planar case is that after going through the diocotron resonance (where the real part of $\omega_{e}=0$ ), this quantity then goes slightly negative, and then begins to turn back toward the resonance. In the planar case, or a low aspect ratio cylindrical case, such never happens, since the drift velocity is always monotonically increasing in magnitude. However, in a high aspect ratio cylindrical case, such as the A6, one can have the magnitude of the drift velocity decreasing after one passes through the region of the sheath. (Mainly because the radial electric field will vary as $r^{-1}$, once one is outside the sheath.) In almost all our solutions, we have seen the real part of $\omega_{e}$ returning toward the resonance, after the sheath has been passed, and in several cases, even crosses zero and has a second resonance, just below the anode. An example of this is shown in Fig. 4. As one can see, there is a second peak in the rf oscillations at the second resonance, and of course, there will be a corresponding secondary boost to the amplitudes of the rf oscillations of the electrons, before they strike the anode.

For other solutions at other ambient magnetic fields, we have seen similar behaviors in the dc density profiles and the rf Lagrangian displacements. Invariably, the lower magnetic fields give a "soft" sheath, which in some cases, can extend out to one-half of the cathode-anode spacing. Furthermore, the magnitude of the Lagrangian displacements become smaller, even at the diocotron resonance, with the value at the anode being even smaller still, only a small fraction of the maximum value at the diocotron resonance. A typical example is shown in Fig. 5. The slackness of the density profile and the lack of significant rf oscillations at the anode would suggest that at these parameters, one should not expect this to be an optimum operating state, and experimentally, it is not. ${ }^{25}$

At the higher magnetic fields, the sheath becomes quite "hard," and almost Brillouin in shape, except for a rather small "shelf" extending out to the anode, as shown in Fig. 6. The harder the density profile becomes, the smaller the attached shelf is, in order to have a consistent solution. It is this relationship that seems to be the dominant limitation on

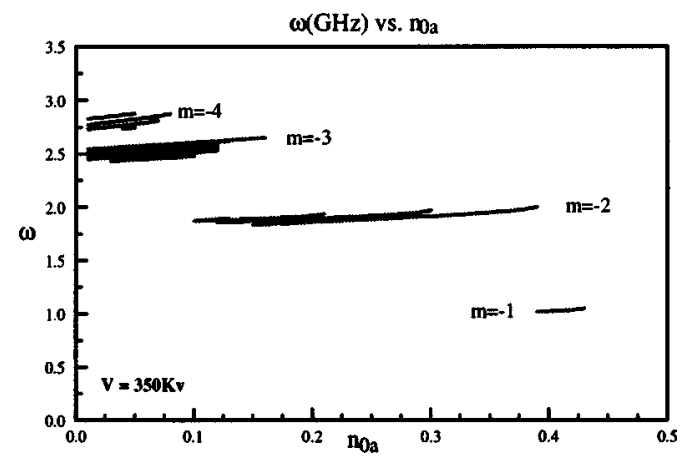

FIG. 7. The eigenfrequencies for $V=350 \mathrm{kV}$. 

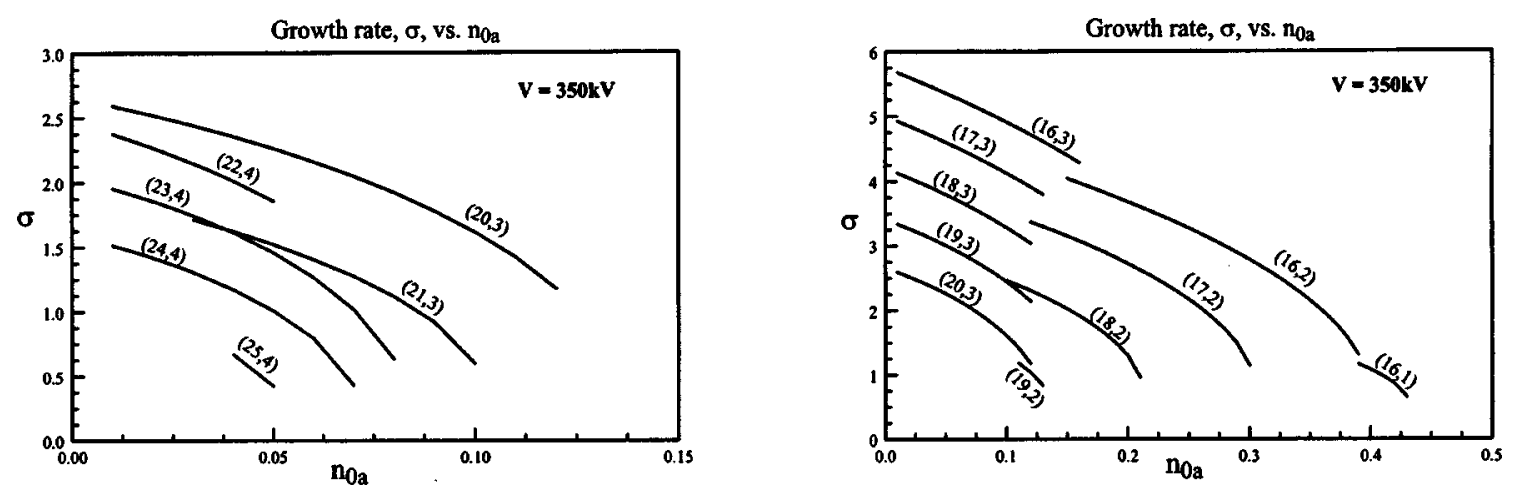

FIG. 8. The growth rate $\sigma$ at $V=350 \mathrm{kV}$ as a function of $n_{0 a}$, at various values of $B_{\text {ext }}$ and the modal number $m$. The quantities in the parentheses are the values of the ambient magnetic field, in $\mathrm{GHz}$, and the negative of the modal number $m$.

the power delivered at the higher magnetic fields. The more tightly the Brillouin sheath is held, the more limited are the rf oscillations. Also, we see in Fig. 6(b) another example of the doubled peaked structure of the Lagrangian displacements. These structures tend to dominate at the higher magnetic fields, and may play a role in delivering higher power levels. However, this range in the A6 is limited. Attempts to find solutions at $V=350 \mathrm{kV}$ and $B_{\text {ext }}=25 \mathrm{GHz}(8.9 \mathrm{KG})$ have been generally unsuccessful, with only a small section of solutions being found, and those are in a parameter range that one would consider to be likely unstable. We also note that $B_{\text {ext }}=25 \mathrm{GHz}$ is well above the optimum operating range for the A6 (see Fig. 23 in Ref. 6), so the lack of solutions in this range is not surprising.

We can compactly summarize the remaining features of these solutions in a series of plots for the operating parameters. In Fig. 7, we show a plot of the eigenfrequencies for the range of the solutions found at $V=350 \mathrm{kV}$. The most obvious feature is a general independence of the parameters $B_{\text {ext }}$ and $n_{0 a}$.

The growth rate $\sigma$ is shown in Fig. 8, for the fastest growing mode. Here, in Fig. 8(a), one sees a consistent pattern wherein the instability, which initiates at the lower values of $n_{0 a}$, starts off as a $\pi$ mode $(m=-3)$, except at the higher magnetic fields, shown in Fig. 8(b), where it starts off as a $m=-4$ mode. However, as the rf amplitude grows, wherein $n_{0 a}$ has to increase, for the lower magnetic fields, the growth rate of the $\pi$ mode eventually becomes exceeded by the growth rate of the $m=-2$ mode, and then at very high $n_{0 a}$ values, even by the $m=-1$, for $B_{\text {ext }}=16 \mathrm{GHz}(5.7$ K G). However, at the higher magnetic fields, no such mode switching occurs, and one has the $m=-4$ mode as being the fastest growing mode. We note that for $B_{\text {ext }}=21 \mathrm{GHz}(7.5$ $\mathrm{K} \mathrm{G}$ ), we were unable to locate a consistent solution for $n_{0 a}$ less than 0.03 .

The magnitude of the $\phi$ component of the electric field at the anode $E_{1 \phi a}$ determines the magnitude of the rf fields in the vane structure, and consequently, the power flow. These solutions are shown in Fig. 9 for $V=350 \mathrm{kV}$, as a function of $n_{0 a}$, at various values of $B_{\text {ext }}$ and the modal number $m$. The quantities in the parentheses are the values of the ambient magnetic field, in $\mathrm{GHz}$, and the negative of the modal number $m$. Note that the sections of the curves with negative slope, corresponds to the situation shown in Fig. 2(d), wherein as one increases $n_{0 a}$, the amplitude of the rf oscillations at the anode decreases. Thus these negative slope sections of the curves are most likely unphysical solutions.

One also sees from here how the characteristics of the dc profile will shift as the rf fields grow. As the device is initiated, the classical Brillouin flow will form. Due to the Rayleigh instability, ${ }^{14}$ the nonlinear diffusion process will initiate, with the Brillouin flow reshaping as shown in the earlier figures. During this reshaping, the rf fields will grow out of the noise, with the mode with the largest growth rate dominating. Those modes with the largest growth rate, are the ones shown in Fig. 8. As the rf mode grows, $E_{1 \phi a}$ must

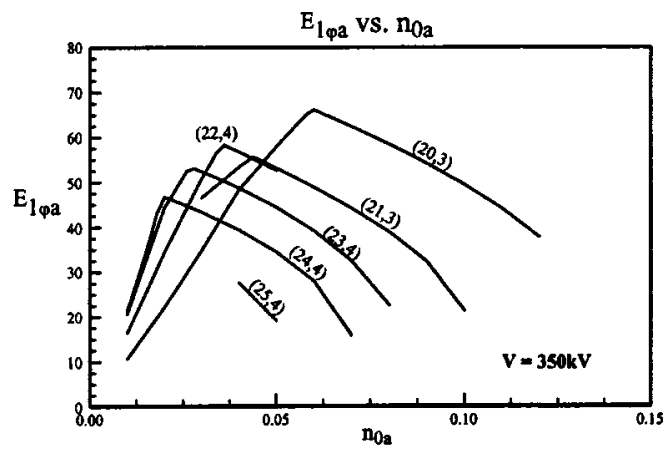

FIG. 9. The $\phi$ component of the magnitude of the electric field at the anode at $V=350 \mathrm{kV}$, as a function of $n_{0 a}$, at various values of $B_{\text {ext }}$ and the modal number $m$. The quantities in the parentheses are the values of the ambient magnetic field, in GHz, and the negative of the modal number $m$. 

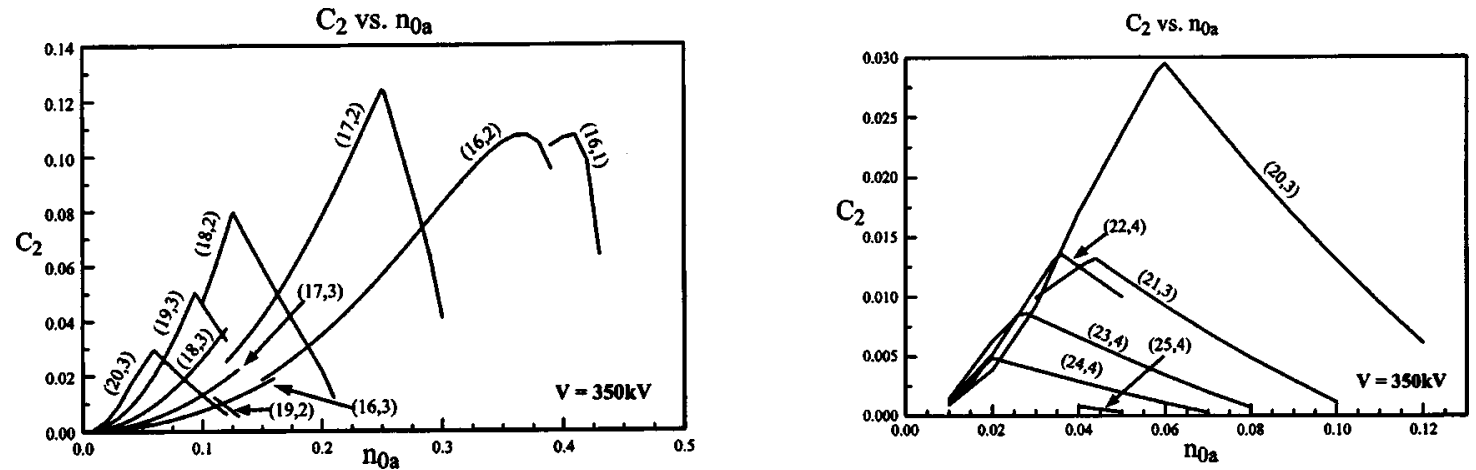

FIG. 10. The values of the coefficient $C_{2}$ at $V=350 \mathrm{kV}$, as a function of $n_{0 a}$, at various values of $B_{\text {ext }}$ and the modal number $m$. The quantities in the parentheses are the values of the ambient magnetic field, in $\mathrm{GHz}$, and the negative of the modal number $m$.

grow at the rate determined by $\sigma$ in Fig. 8. Thus in Fig. 9, we see that $n_{0 a}$ must then increase to match to the increasing amplitude of the rf oscillations in the SWS. However, once $E_{1 \phi a}$ reaches its maximum amplitude, it can grow no further, and the device then enters into what we have called the "saturation stage." 20 This is an operating state wherein there is no growth, and the device steadily delivers power. The study of the stability of this saturation stage is still to be done.

The coefficient $C_{2}$ for $V=350 \mathrm{kV}$, as a function of $n_{0 a}$, at various values of $B_{\text {ext }}$ and the modal number $m$, is shown in Fig. 10. This coefficient is essentially the second-order dc radial velocity, in $\mathrm{cm} / \mathrm{ns}$, and gives how rapidly the electrons transit from the cathode to the anode.

There is another feature of these results that needs to be mentioned. As one can observe from Figs. 8(a) and 9(a), at a fixed $B_{\text {ext }}$, there are overlaps in the domains of the $m$ $=-2$ and $m=-3$ modes. It is in the neighbor of these domains of $n_{0 a}$ values, that one could expect mode competition to occur, since fluctuations in the background density could throw the device from one mode to another, or even allow both to coexist. Note that such would not occur for magnetic fields greater than about $18.5 \mathrm{GHz}$, since the mode switching would then occur after $E_{1 \phi a}$ has peaked.

Another parameter of interest is the ratio of the secondorder radial velocity $v_{2 r}$ compared to the amplitude of the radial rf velocity $\left|v_{1 r}\right|$. This ratio can indicate the possibility of an instability in the saturation stage. For the nonrelativistic planar case, it has been shown in Ref. 20 that the saturation stage will have no consistent solution, if at some value of $r, v_{2 r} \geqslant\left|v_{1 r}\right|$. In fact, the instability that would then occur, would have to be one in which the solution becomes nonstationary, and very likely, chaotic. For comparison, from our unpublished numerical data on the initiation stage of the nonrelativistic $\mathrm{T} 266$, the ratio of $v_{2 y} /\left|v_{1 y}\right|$ is never seen to become larger than 0.25 . This device is a CFA and does dependably amplify an rf wave. For the A6, and in particular for the data in the first panel in Fig. 11, one notes that at about $r=1.64 \mathrm{~cm}$, this ratio has a value of $\approx 0.55$. While this ratio is below unity, nevertheless it is significantly larger than that for the T266. Furthermore, should there happen to be any strong fluctuations in these velocity components, the equilibrium value of this ratio could be potentially pushed above unity, at which point, the stationary solution would go unstable, and the device would shut down. This is indeed an observation feature of the $\mathrm{A} 6,{ }^{33}$ and this could be a possible explanation as to why it occurs.

In the second panel of Fig. 11, we show the maximum amplitudes of the $\phi$ component of the electric field at the anode, $E_{1 \phi a}$, which were taken from the peak values in Fig. 9 , as a function of $B_{\text {ext }}$. These values show what the operating modes are, just before the system continues on into the saturation stage. One notes here that the $m=-2$ mode dominates at lower values of $B_{\text {ext }}$, shifts to the $m=-3$ mode as
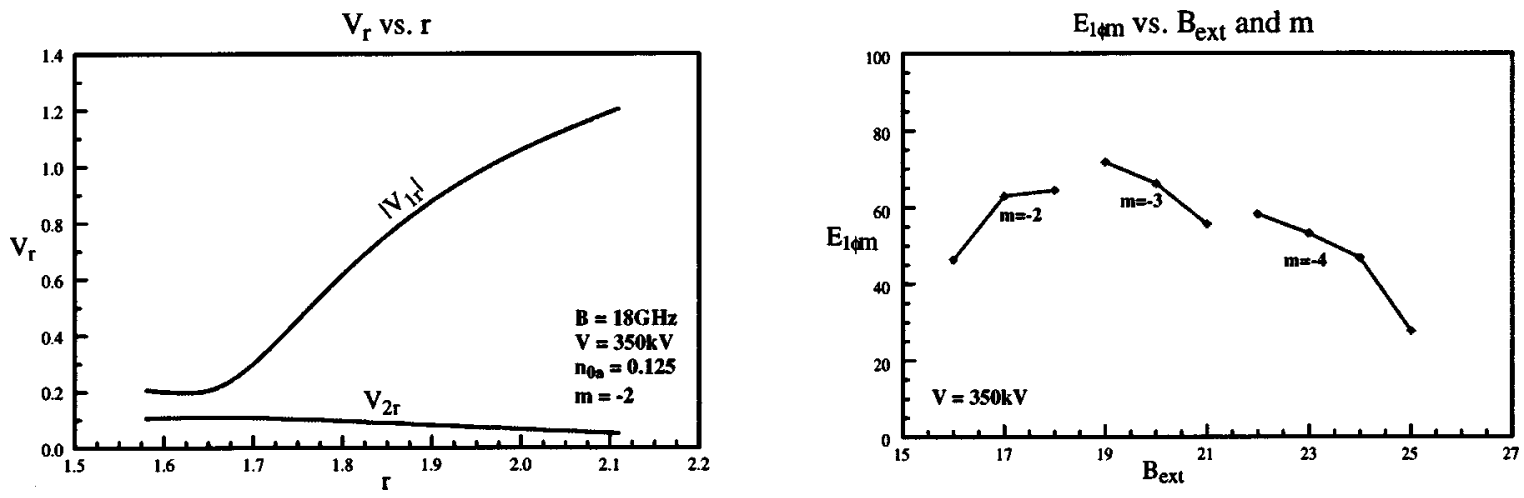

FIG. 11. In the first panel, we show second-order radial velocity and the magnitude of the rf radial velocity, at $V=350 \mathrm{kV}$, as a function of $r$. Near the cathode, at the left, the smallest value of the ratio of $v_{2 r} /\left|v_{1 r}\right|$ is $\approx 0.55$. In the second panel, we show $E_{1 \phi m}$, which is the maximum amplitude of $E_{1 \phi a}$ (obtained from the peak values in Fig. 9), as a function of $B_{\text {ext }}$. 

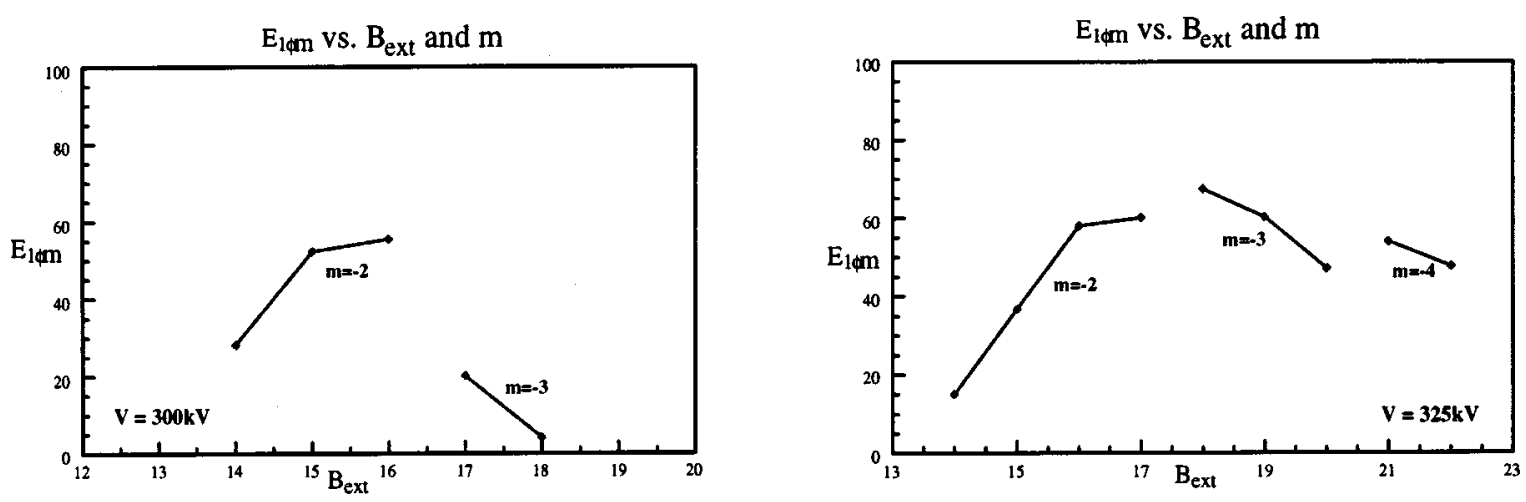

FIG. 12. The maximum amplitude of $E_{1 \phi a}$ for 300 and $325 \mathrm{kV}$ as a function of $B_{\text {ext }}$.

$B_{\text {ext }}$ increases, and finally shifts to the $m=-4$ mode at the higher values of $B_{\text {ext }}$. From this curve, one sees that the device would initiate when $B_{\text {ext }}$ is in the range of 17-24 $\mathrm{GHz}$, or 6-9 K G, which is exactly where the A6 does operate at $350 \mathrm{kV} .^{25}$ We have also studied the initiation of the A6 at other voltages as well. In general, the results are similar to those for $350 \mathrm{kV}$, however, with expected shifts in the various parameters. For brevity at these other voltages, we will only show the curves corresponding to the second panel in Fig. 11, which will indicate the corresponding operating ranges for $B_{\text {ext }}$. In Fig. 12, we show the results for 300 and $325 \mathrm{kV}$. What one observes here is that at $300 \mathrm{kV}$, the values of $E_{1 \phi m}$ are considerably lower than those at 325 and 350 $\mathrm{kV}$. In fact, the values for $E_{1 \phi m}$ for the $\pi$ mode appear to be so low that one would expect the device to certainly not operate in the $\pi$ mode, and perhaps not at all. On the other hand, in the second panel, at $325 \mathrm{kV}$, one sees a dramatic increase in the presence of the $\pi$ mode, and thus would expect the device to operate in that mode, although at a lower power than at $350 \mathrm{kV}$.

In Fig. 13, we show the results for 400 and $500 \mathrm{kV}$. What one observes here is that at $400 \mathrm{kV}$, the device should operate in the range of $18-24 \mathrm{GHz}$, while at $500 \mathrm{kV}$, it should operate in the range of $21-28 \mathrm{GHz}$. We have not investigated our model above $500 \mathrm{kV}$.

In a recent study, ${ }^{34}$ numerical PIC simulations have been performed for a "rising-sun" magnetron operating at approximately $500 \mathrm{kV}$, where it was found that mode competition was a major problem in the operation of a rising-sun magnetron at the higher voltages. We have already remarked that mode competition could be expected to occur, whenever the domains of the $m=-3$ and $m=-2$ overlap, as seen in Fig. 8. From the corresponding curve at $500 \mathrm{kV}$, the domains of the $m=-3$ and $m=-2$ mode do overlap below about 25 $\mathrm{GHz}$, above which only the $\pi$ mode exists. However, we do not observe a significant increase in the widths of the overlap domains between 350 and $500 \mathrm{kV}$.

\section{CONCLUDING REMARKS}

Using only two modes, we have analytically modeled the initiation stage of an A6 relativistic magnetron. The results obtained show reasonably good quantitative agreement with the known operating range of the device. This analysis gives one an understanding of the important physical processes in the device, and allows one to understand the nature of the solutions for the various variables and parameters. One new feature is that in a cylindrical device, with a sufficiently large aspect ratio, the electrons can be given a second "boost" on their way to the anode (see Fig. 4), where the diocotron resonance occurs not only at the edge of the Brillouin sheath, but also just under the anode. This should be a feature of high aspect ratio cylindrical systems in general. All that is required for a double diocotron resonance to occur in a cylindrical device is for the aspect ratio to be sufficiently large, so that $\omega_{e}$ would recross zero near the anode.

A second observation is that we have a possible mechanism for the random shutting down of such a device. ${ }^{33}$ Per
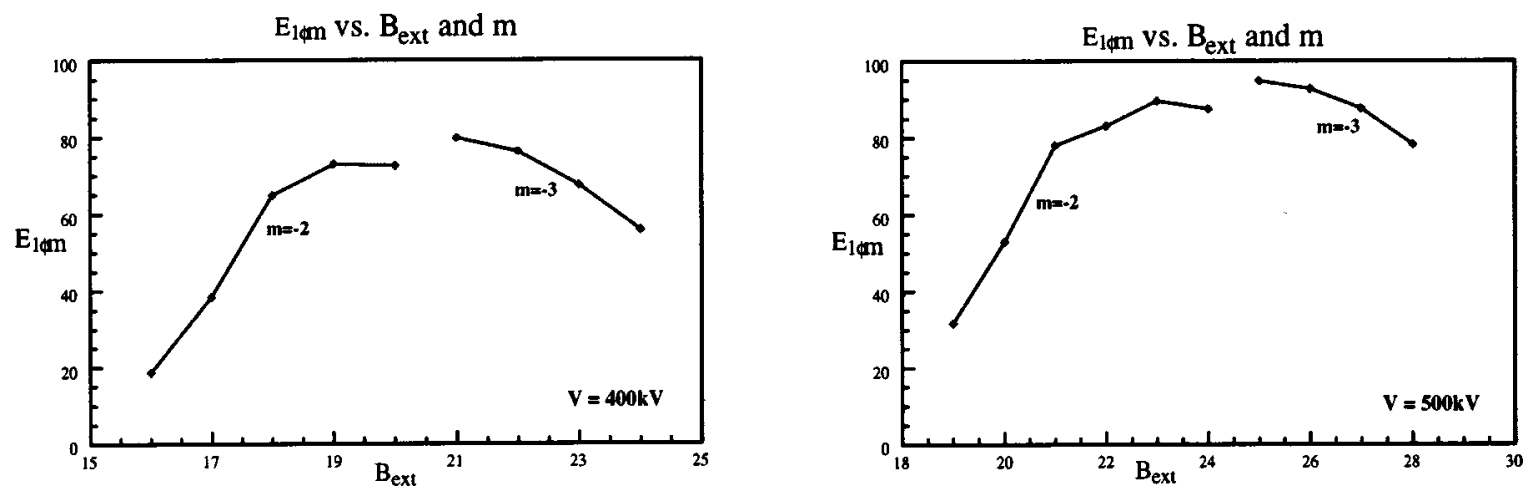

FIG. 13. The maximum amplitude of $E_{1 \phi a}$ for 400 and $500 \mathrm{kV}$ as a function of $B_{\mathrm{ext}}$. 
previous results for the nonrelativistic, planar model, we had shown that a too large of a cathode-anode current would drive the background density unstable, and thereby destroy the steady delivery of power. $^{20}$ The necessary criteria for stability was found to be that the radial dc velocity must always be less than the magnitude of the radial rf velocity. Although this is a nonrelativistic result, one would expect the same criteria to also apply in the relativistic case, with small corrections. For our model of the A6, we found that the maximum ratio of the dc radial velocity to the rf radial velocity could become as large as 0.55 . While this is still smaller than unity, for the nonrelativistic T266, the maximum ratio was always less than 0.25 . Thus the A6 operates closer to this instability limit than the T266 does. Should there happen to be random fluctuations in the A6 that would be sufficiently large near the edge of the sheath, then one could expect them to randomly turn on this instability. The key question then becomes, just how smooth and steady are these velocity flows in the actual device? Per PIC code results, as in Fig. 25 of Ref. 6, one suspects that random fluctuations of this size may not be unusual. If so, then this could well be an explanation as to why the A6 would tend to shut down, after entering upon the saturation stage.

\section{ACKNOWLEDGMENTS}

The author acknowledges valuable assistance by Dr. Jian-ping $\mathrm{Gu}$ and Irina Polandova in performing many of the numerical computations, and composing plots of the results.

This research has been supported in part by the Air Force Office of Scientific Research.

\footnotetext{
${ }^{1}$ A. Palevski, G. Bekefi, and A. Drobot, J. Appl. Phys. 52, 4938 (1981).

${ }^{2}$ G. E. Dombrowski, IEEE Trans. Electron Devices 35, 2060 (1988).

${ }^{3}$ S. Riyopoulos, Phys. Rev. E 47, 2839 (1993).

${ }^{4}$ R. W. Lemka, T. C. Genoni, and T. A. Spencer, Phys. Plasmas 6, 603 (1999).

${ }^{5}$ R. C. Davidson, Phys. Fluids 27, 1804 (1984).
}

${ }^{6}$ R. C. Davidson, H.-W. Chan, C. Chen, and S. Lund, Rev. Mod. Phys. 63, 341 (1991).

${ }^{7}$ H.-W. Chan, C. Chen, and R. C. Davidson, J. Appl. Phys. 73, 7053 (1993).

${ }^{8}$ S. Riyopoulos, Phys. Plasmas 3, 1137 (1996).

${ }^{9}$ D. J. Kaup and G. E. Thomas, Stud. Appl. Math. 81, 37 (1989); 83, 271(E) (1990).

${ }^{10}$ D. J. Kaup and G. E. Thomas, Phys. Plasmas 3, 771 (1996).

${ }^{11}$ D. J. Kaup and G. E. Thomas, J. Plasma Phys. 58, 145 (1997).

${ }^{12}$ D. J. Kaup and G. E. Thomas, J. Plasma Phys. 59, 259 (1998).

${ }^{13}$ L. Brillouin, Phys. Rev. 67, 260 (1945).

${ }^{14}$ P. G. Drazin and W. H. Reid, Hydrodynamic Stability (Cambridge University Press, New York, 1981) Chap. 4.

${ }^{15}$ R. C. Davidson, Phys. Fluids 28, 1937 (1985).

${ }^{16}$ R. C. Davidson and K. T. Tang, Phys. Fluids 28, 1169 (1985).

${ }^{17}$ R. C. Davidson, K. T. Tsang, and J. A. Swegle, Phys. Fluids 27, 2332 (1984).

${ }^{18}$ D. J. Kaup, T. I. Lakoba, and G. E. Thomas, Proc. SPIE 3158, 137 (1997).

${ }^{19}$ D. J. Kaup, Proc. SPIE 4371, 49 (2001).

${ }^{20}$ D. J. Kaup, Proc. SPIE 4720, 67 (2002).

${ }^{21}$ V. M. Ayres, H. C. Chen, R. A. Stark, H. S. Uhm, and H. E. Brandt, Phys. Fluids B 4, 3396 (1992).

${ }^{22}$ S. Riyopoulos, Phys. Plasmas 6, 1344 (1999).

${ }^{23}$ D. J. Kaup, Phys. Plasmas 8, 2473 (2001).

${ }^{24}$ D. J. Kaup and G. E. Thomas, Proc. SPIE 4031, 54 (2000).

${ }^{25}$ A. Palevski and G. Bekefi, Phys. Fluids 22, 986 (1979).

${ }^{26} \mathrm{~J}$. Swegle and E. Ott, Phys. Fluids 24, 1821 (1981).

${ }^{27}$ O. Buneman, R. H. Levy, and L. M. Linson, J. Appl. Phys. 37, 3203 (1966).

${ }^{28}$ T. M. Antonsen, Jr., E. Ott, C. L. Chang, and A. T. Drobot, Phys. Fluids 28, 2878 (1985).

${ }^{29}$ D. J. Kaup, Phys. Fluids B 2, 2253 (1990).

${ }^{30}$ H. S. Uhm, Phys. Fluids B 4, 740 (1992).

${ }^{31}$ MACSYMA, a symbolic computational software that is no longer currently being sold. However, the same computations done by us could also be done on most of the other currently available symbolic computational software.

${ }^{32}$ Strictly speaking, $E_{2 \phi}$ need not vanish in the slots, but because we are assuming the dc mode to be $\phi$ independent, since it must vanish on the vanes, then it must also vanish at the slots.

${ }^{33}$ M. R. Lopez, R. M. Gilgenbach, Y. Y. Lau, D. W. Jordan, M. D. Johnston, M. C. Jones, V. B. Neculaes, T. A. Spencer, J. W. Luginsland, M. D. Haworth, R. W. Lemke, D. Price, and L. Ludeking, Proc. SPIE 4720, 10 (2002).

${ }^{34}$ R. W. Lemka, T. C. Genoni, and T. A. Spencer, Phys. Plasmas 7, 706 (2000). 